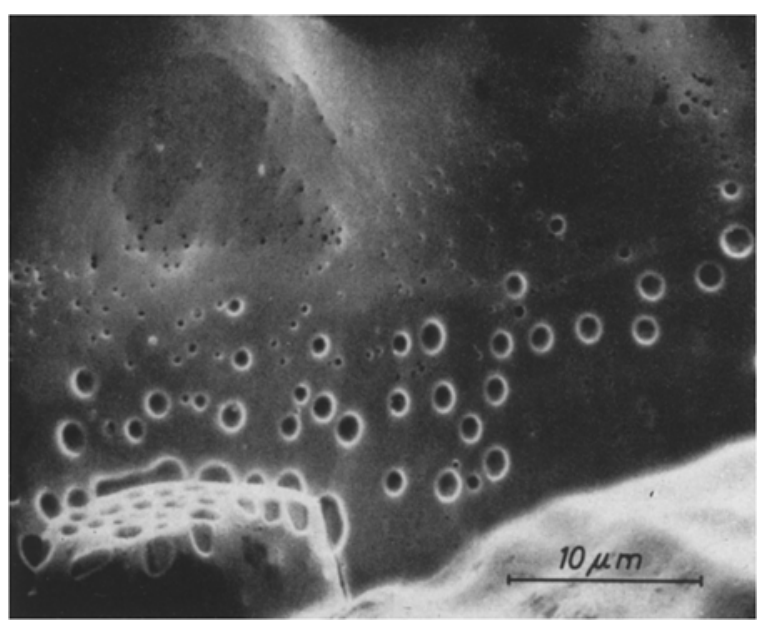

Fig. 1. Rasterelektronenmikroskopische Aufnahme der Bruch fläche eines Bor-gedopten Molybdändrahtes von 0,6 mm Durch messer, $15 \mathrm{~min}$ bei $1900^{\circ} \mathrm{C}$ geglüht

Präparation wurde der etwa $83 \mathrm{ppm}$ Bor enthaltende Molyb dändraht $(\varnothing 0,6 \mathrm{~mm})$ bei $1900{ }^{\circ} \mathrm{C} 15 \mathrm{~min}$ geglüht, dann unter flüssigem Stickstoff gebrochen und die Bruchfläche im REM bei $20 \mathrm{kV}$ Beschleunigungsspannung untersucht. Die Drahtprobe war bevorzugt interkristallin gebrochen, nur vereinzelt trat transkristalliner Spaltbruch auf. An den Korngrenzen in der Nähe der Drahtoberfläche konnten Bläschen von etwa $1-2 \mu \mathrm{m}$ Durchmesser beobachtet werden. Die Bläschengröße nimmt mit der Entfernung von der Drahtoberfläche rasch ab, sie beträgt bei etwa. $20 \mu \mathrm{m}$ ungefähr $5000 \AA$ und ist im Drahtinneren kleiner als die Auflösungsgrenzen des Rasterelektronen mikroskopes (etwa $200 \AA$ ). Der Einflu $\beta$ so kleiner Poren auf die Versetzungsbewegung bei plastischer Verformung und auf den Rekristallisationsvorgang ist sebr ausgeprägt [1] und dürfte auch die Wirkung feinster fester Dispersionen übertreffen [2]. Dies äußert sich im Sintermolybdän auch bei sehr kleinen Borgehalten in einer starken Kornwachstumshemmung, in einer gerichteten Sekundär-Rekristallisation und in einer Erhöhung der Kriechfestigkeit des Reinmetalles. Eine entsprechende Wirkung von Mikrolegierungszusätzen an Bor ist auch bei Wolfram nachgewiesen worden [3] und bei anderen kubisch raumzentrierten Metallen wie Tantal, Niob, Vanadium u.a. zu erwarten. Bei anderen Metallen könnten Gasbläschen bildende Dopmittel ähnlich wirken, wobei die Stabilität der Bläschen und ihr Thermodiffusionsverhalten ein Kriterium für ihre Wirksamkeit sind.

Eingegangen am 22. November 1973

1. Moon, D. M., Stickler, R.: Phil. Mag. 24, 1087 (1971)

2. Moon, D. M., Koo, R. C.: Met. Trans, 2, 2115 (1971)

3. Miliner, T., Tury, P.: U.S.Pat, 2,012825 (1935)

\section{Das erste Oxoberyllat mit Inselstruktur: $\mathrm{K}_{4}\left[\mathrm{Be}_{2} \mathrm{O}_{4}\right]$}

P. Kastner [1] und R. Hoppe

Institut für Anorganische und Analytische Chemie der Universität Gießen

Über Oxoberyllate ist trotz der lange bekannten Amphoterie von $\mathrm{Be}(\mathrm{OH})$, wenig bekannt. Nach Pulverdaten existieren $\mathrm{Na}_{4} \mathrm{BeO}_{3}$ [2] und $\mathrm{Li}_{2} \mathrm{Be}_{2} \mathrm{O}_{3}$ [3], beide mit unbekannter Struktur.

Neu erhielten wir $\mathrm{K}_{2} \mathrm{BeO}_{2}$ in Form quaderfötmiger, farbloser Einkristalle $\left[\mathrm{KO}_{0,54} / \mathrm{BeO}, \mathrm{K}: \mathrm{Be}=2,2: 1 ; 600^{\circ} \mathrm{C}, 7 \mathrm{~d}, \mathrm{Ni}\right.$ Bömbchen], die nach Drehkristall-, Weissenberg- und Präzessionsaufnahmen monoklin, $C_{2 h}^{5}-P 2_{1} / b$ [4], mit

$a=7,09_{8}, b=10,57_{7}, c=5,70_{6} \AA, \gamma=131,3^{\circ}[4], Z=4$

kristallisieren; $d_{\mathrm{pyk}}=2,5, a_{\mathrm{rO}}=2,46 \mathrm{~g} \cdot \mathrm{cm}^{-3}$.

Die Kristallstruktur 1675 von 711 möglichen hho-hh4, 2-Kreisdiffraktometerdaten (Fa. Stoe, Stadi-2), Mo- $K_{\alpha}$, isotrope
Tabelle 1. Lageparameter und $B$-Werte aller Teilchen von $\mathrm{K}_{2} \mathrm{BeO}_{2}$ (in Klammern die Standardabweichungen, bezogen auf die letzten Stellen)

\begin{tabular}{llllll}
\hline $\begin{array}{l}\text { Teil- } \\
\text { chen }\end{array}$ & $\begin{array}{l}\text { Punkt- } x \\
\text { lage }\end{array}$ & $y$ & $z$ & $B\left[\AA^{2}\right]$ \\
\hline $\mathrm{K}^{\prime}$ & $4 e$ & $0,3558(1)$ & $0,26069(8)$ & $0,0643(2)$ & $1,74(2)$ \\
$\mathrm{K}^{\prime \prime}$ & $4 e$ & $0,0269(1)$ & $0,38952(8)$ & $0,1633(2)$ & $1,51(2)$ \\
$\mathrm{Be}$ & $4 e$ & $0,6500(6)$ & $0,0251(5)$ & $0,0859(9)$ & $1,3(1)$ \\
$\mathrm{O}^{\prime}$ & $4 e$ & $0,5078(4)$ & $0,0908(3)$ & $0,1379(5)$ & $1,83(7)$ \\
$\mathrm{O}^{\prime \prime}$ & $4 e$ & $0,8650(4)$ & $0,0598(3)$ & $0,2035(5)$ & $1,90(8)$ \\
\hline
\end{tabular}

Tabelle 2. Alle interatomaren $A$ bstände bis $4,0 \AA$ bei $\mathrm{K}_{2} \mathrm{BeO}_{2}$

\begin{tabular}{|c|c|c|c|c|c|}
\hline & $K^{\prime}$ & $\mathrm{K}^{\prime \prime}$ & $\mathrm{Be}$ & $O^{\prime}$ & $\mathrm{O}^{\prime \prime}$ \\
\hline$K^{\prime}$ & $3,604(2 \times)$ & $\begin{array}{l}3,073 \\
3,345 \\
3,438 \\
3,631 \\
3,986\end{array}$ & $\begin{array}{l}2,909 \\
3,116 \\
3,565 \\
3,757 \\
3,854\end{array}$ & $\begin{array}{l}2,670 \\
2,705 \\
3,358 \\
3,480\end{array}$ & $\begin{array}{l}2,742 \\
3,045 \\
3,121 \\
3,772\end{array}$ \\
\hline $\mathbf{K}^{\prime \prime}$ & $\begin{array}{l}3,073 \\
3,345 \\
3,438 \\
3,631 \\
3,986\end{array}$ & $\begin{array}{l}3,203 \\
3,938(2 \times)\end{array}$ & $\begin{array}{l}2,934 \\
3,026 \\
3,768 \\
3,911\end{array}$ & $\begin{array}{l}2,808 \\
2,867\end{array}$ & $\begin{array}{l}2,686 \\
2,806 \\
2,872 \\
3,136\end{array}$ \\
\hline $\mathrm{Be}$ & $\begin{array}{l}2,909 \\
3,116 \\
3,565 \\
3,757 \\
3,854\end{array}$ & $\begin{array}{l}2,934 \\
3,026 \\
3,768 \\
3,911\end{array}$ & 2,070 & $\begin{array}{l}1,586 \\
1,605\end{array}$ & $\begin{array}{l}1,475 \\
3,545 \\
3,994\end{array}$ \\
\hline $\mathrm{O}^{\prime}$ & $\begin{array}{l}2,670 \\
2,705 \\
3,358 \\
3,480\end{array}$ & $\begin{array}{l}2,808 \\
2,867\end{array}$ & $\begin{array}{l}1,586 \\
1,605\end{array}$ & 2,429 & $\begin{array}{l}2,788(2 \times) \\
3,907\end{array}$ \\
\hline $\mathrm{O}^{\prime \prime}$ & $\begin{array}{l}2,742 \\
3,045 \\
3,121 \\
3,772\end{array}$ & $\begin{array}{l}2,686 \\
2,806 \\
2,872 \\
3,136\end{array}$ & $\begin{array}{l}1,475 \\
3,545 \\
3,994\end{array}$ & $\begin{array}{l}2,788(2 \times) \\
3,907\end{array}$ & 3.724 \\
\hline
\end{tabular}

Temperaturfaktoren, keine Absorptionskorrektur, $R=6,26 \%$, $\left.R^{\prime}=7,1, \%\right]$ zeigt, daB unerwartet planare, zweikemige isolierte Baugruppen $\left[\mathrm{BeO} / \mathrm{O}_{2} / 2\right]_{2}$ vorliegen; $\mathrm{Be}^{2+}$ hat gegen $\mathrm{O}^{2-}$ die Koordinationszahl 3. Zwei solcher Dreiecke haben jeweils eine gemeinsame Kante (besetzte Punktlagen, Parameter und Abstände s. Tabelle 1 und 2). Die Koordinationszahl von $\mathrm{K}^{+}$ist deutlich verschieden: $\mathrm{K}^{\prime+}$ hat $3+2, \mathrm{~K}^{\prime \prime}+$ hat $5+1 \mathrm{O}^{2-}$ als Nachbarn. Der Madelung-Anteil der Gitterenergie, MAPLE, ist für $\mathrm{K}_{\mathrm{g}} \mathrm{BeO}_{2}$ (1939,7 $\left.\mathrm{kcal} / \mathrm{Mol}\right)$ praktisch gleich den Werten für die Summe der binären Oxide $\left(\mathrm{K}_{2} \mathrm{O}+\mathrm{BeO}[5]=599,9+\right.$ $1321,6=1921,5 \mathrm{kcal} / \mathrm{Mol}$ )

$\mathrm{Rb}_{2} \mathrm{BeO}_{2}$ ist nach Einkristalldaten mit $\mathrm{K}_{4}\left[\mathrm{Be}_{2} \mathrm{O}_{4}\right]$ isotyp.

Wir danken der Deutschen Forschungsgemeinschaft für die Unterstützung mit Sachmitteln.

Eingegangen am 4. Dezember 1973

1. Teil der Dissertation P. Kastner, Universität Gießen

2. Zintl, E., Morawietz, W.: Z. anorg, allg. Chem. 236, 372 (1938)

3. Turner, P. P., Bartram, S. F.: Inorg. Chem. 6, 833 (1967)

4. Aus apparativen Gründen wählten wir diese Aufstellung

5. Smith, D. K., Newkirk, H. W., Kahn, J. S.: J. electrochem. Soc. 111, $78(1964)$

\section{Identifizierung und Metabolisierbarkeit von polychlorierten Biphenylen}

E. Schulte und L. Acker

Institut für Lebensmittelchemie der Universität Münster

Bei der gaschromatographischen Auftrennung polychlorierter Biphenyle (PCB) hat die Verwendung von Kapillarsäulen einen wesentlichen Fortschritt gebracht [1, 2]. In einem in der Umwelt ubiquitären PCB-HandeIspräparat mit $60 \%$ 